\title{
TITLE:
}

\section{An exact algorithm for the Boolean connectivity problem for $\mathrm{k}$-CNF}

\section{$\operatorname{AUTHOR}(\mathrm{S}):$}

Makino, Kazuhisa; Tamaki, Suguru; Yamamoto, Masaki

\section{CITATION:}

Makino, Kazuhisa ...[et al]. An exact algorithm for the Boolean

connectivity problem for k-CNF. Theoretical Computer Science 2011, 412(35): 4613-4618

\section{ISSUE DATE:}

2011-08

URL:

http://hdl.handle.net/2433/145991

\section{RIGHT:}

(C) 2011 Elsevier B.V.; This is not the published version. Please cite only the published version.; この論文は出版社版でありません。引用の際に は出版社版をご確認ご利用ください。 


\title{
An Exact Algorithm for the Boolean Connectivity Problem for $k$ - $\mathrm{CNF}^{\text {出 }}$
}

\author{
Kazuhisa Makino \\ Graduate School of Information Science and Technology, University of Tokyo \\ Suguru Tamaki \\ Graduate School of Informatics, Kyoto University \\ Masaki Yamamoto \\ Dept. of Informatics, Kwansei-Gakuin University
}

\begin{abstract}
We present an exact algorithm for a PSPACE-complete problem, denoted by CONN $k$ SAT, which asks if the solution space for a given $k$-CNF formula is connected on the $n$-dimensional hypercube. The problem is known to be PSPACE-complete for $k \geq 3$, and polynomial solvable for $k \leq 2$ [6]. We show that CONNkSAT for $k \geq 3$ is solvable in time $O\left(\left(2-\epsilon_{k}\right)^{n}\right)$ for some constant $\epsilon_{k}>0$, where $\epsilon_{k}$ depends only on $k$, but not on $n$. This result is considered to be interesting due to the following fact shown by [5]: QBF-3-SAT, which is a typical PSPACE-complete problem, is not solvable in time $O\left((2-\epsilon)^{n}\right)$ for any constant $\epsilon>0$, provided that the SAT problem (with no restriction to the clause length) is not solvable in time $O\left((2-\epsilon)^{n}\right)$ for any constant $\epsilon>0$.
\end{abstract}

Keywords:

exponential-time algorithms, Boolean connectivity, CNF satisfiability

\footnotetext{
${ }^{\sqrt{4}}$ A preliminary version of this paper appeared in the Proceedings of the 13th International Conference on Theory and Applications of Satisfiability Testing (SAT 2010), LNCS 6302, pp. 172-180, 2010.

Email addresses: makino@mist.i.u-tokyo.ac.jp (Kazuhisa Makino), tamak@kuis.kyoto-u.ac.jp (Suguru Tamaki), masaki.yamamoto@kwansei.ac.jp (Masaki Yamamoto)
} 


\section{Introduction}

There are so many NP-hard problems around the world, which are considered to be intractable. To deal with those intractable problems, efficient algorithms with good approximation ratio or working well on average, have been proposed. Another approach to dealing with intractable problems is to develop algorithms that exactly solve the problems, so-called exact algorithms, where exact algorithms usually run in super-polynomial time, but exponentially faster than trivial ones. See $[20,17]$ for surveys on this topic. A number of exact algorithms for typical NP-hard problems have been proposed, and novel techniques for bounding the running time have been found: E.g., $[1,7,2]$ for the traveling salesman problem, $[4,9,3]$ for graph partitioning problems such as the graph coloring problem, and [14, 13, 16, 8, 15] for the satisfiability problem.

Viewing this approach in terms of computational complexity, we are concerned with the following question: Given an NP-hard problem of solution length $n$ or witness length $n$, (for example, $n$ denotes the number of vertices of a graph for the traveling salesman problem, or the number of variables of a formula for the satisfiability problem) is there an exact algorithm for the problem in time $O\left(2^{n}\right)$, or $O\left((2-\epsilon)^{n}\right)$ for some constant $\epsilon>0$ ? Here, we assume that the length of instances with solution length $n$ or witness length $n$ is bounded by a polynomial in $n$. Moreover, as usual, we omit the polynomial factor in the $O$-notation when concerning with an upper bound of exponential-time.

The oldest result for this kind of questions is for the traveling salesman problem by Bellman [1] and by Held and Karp [7]. Given an undirected graph $G=(V, E)$ and a length function $\ell: E \rightarrow R_{+}$, the problem asks for finding a shortest Hamilton cycle. It is easy to see that the problem is solvable in time $O(n !)$. However, it is indeed not so easy to see that it is solvable in time $O\left(2^{n}\right)$. These two papers $[1,7]$ gave an affirmative answer to this question. There are several results that give such an affirmative answer: for example, $[4,9]$ for the $k$-coloring problem showed that it is solvable in time $O\left(2^{n}\right)$ for any $k$ (not necessarily constant) while it is trivially solvable in time $O\left(k^{n}\right)$, and [19] for the maximum satisfiability problem where the clause length of an instance is at most two showed that it is solvable in time $O\left(1.731^{n}\right)$ while it is trivially solvable in time $O\left(2^{n}\right)$.

One of the most notable questions of this kind, which are still open, is for the satisfiability problem (SAT). This problem asks if there is a satisfying 
assignment for a given conjunctive normal form (CNF) formula $\varphi$ with no restriction to the clause length. It is clear that the problem is solvable in time $O\left(2^{n}\right)$. However, it is still open whether it is solvable in time $O\left((2-\epsilon)^{n}\right)$ for some constant $\epsilon>0$. Another well-known open question is to ask whether the traveling salesman problem is solvable in time $O\left((2-\epsilon)^{n}\right)$ for some constant $\epsilon>0$.

While developing exact algorithms for NP-complete problems and their optimization problems, we rarely see exact algorithms for decision problems in complexity classes beyond NP, such as the second and higher levels of PH, PSPACE, EXP, etc. There is one exceptional problem as far as we know: the quantified Boolean formula (QBF) problem, that is a typical PSPACEcomplete problem, even if given Boolean formulas are restricted to 3-CNF. Williams [18] proposed an exact algorithm for this problem. However, he analyzed the running time with respect to the number of clauses, but not the number of variables. (Apart from decision problems, there are several problems solvable in time $O\left((2-\epsilon)^{n}\right)$, e.g., \#k-SAT problem, which is \#Pcomplete for $k \geq 2$. For this problem, we easily obtain an $O((2-\epsilon))^{n}$-time exact algorithm, by using a simple backtracking algorithm for $k$-SAT. The best upper bound for \#3-SAT, for example, can be found in [10].)

In this paper, we show that the following PSPACE-complete problem, denoted by CONNkSAT, is solvable in time $O\left((2-\epsilon)^{n}\right)$ for $n$ variables: given a $\mathrm{k}-\mathrm{CNF}$ formula $\varphi$ over $n$ variables, decide whether the solution space of $\varphi$ is connected on the $n$-dimensional hypercube. (See the next section for the precise definition.) This problem was proposed by Gopalan, Kolaitis, Maneva, and Papadimitriou to investigate connectivity properties on Boolean formulas. It is known that the problem is PSPACE-complete for $k \geq 3$, while it is in $\mathrm{P}$ for $k \leq 2$ [6]. Moreover, it is known to be coNP-complete, if given formulas are restricted to Horn $3-\mathrm{CNF}$ [12]. We show that CONN $k$ SAT for $k \geq 3$ is solvable in time $O\left(\left(2-\epsilon_{k}\right)^{n}\right)$ for some constant $\epsilon_{k}>0$, where $\epsilon_{k}$ depends only on $k$, but not on $n$. It seems to be the first nontrivial result that gives an $O\left((2-\epsilon)^{n}\right)$-time algorithm for a certain PSPACE-complete problem in terms of the number of variables. Furthermore, this result is considered to be interesting because Calabro, Impagliazzo, and Paturi [5] recently showed the following fact on $\Pi_{2}-3-\mathrm{SAT}$ : this problem, which is a typical $\Pi_{2}^{P}$-complete problem, is the $\mathrm{QBF}$ problem over 3-CNF formulas, where the quantifier starts with $\forall$, and the number of changes between two types of consecutive quantifiers is at most one. They showed that $\Pi_{2}-3-\mathrm{SAT}$ is not solvable in time $O\left((2-\epsilon)^{n}\right)$ for any constant $\epsilon>0$, provided that the 
SAT problem (with no restriction to the clause length) is not solvable in time $O\left((2-\epsilon)^{n}\right)$ for any constant $\epsilon>0$. It means that the (general) QBF over 3CNF formulas, which is a typical PSPACE-complete problem, is not solvable in time $O\left((2-\epsilon)^{n}\right)$ for any constant $\epsilon>0$ under the same assumption.

\section{Preliminaries}

In this paper, we deal with $k$-CNF formulas, where the length of each clause of a formula is at most $k$. Let $X=\left\{x_{1}, \ldots, x_{n}\right\}$ be a set of Boolean variables. An assignment to $X$ is an element of $\{0,1\}^{n}$. A partial assignment to $X$ is an element of $\{0,1, *\}^{n}$, where we regard a variable assigned $*$ as unassigned. We alternately express partial assignments by pulling out coordinates assigned 0 or 1 , e.g., a partial assignment $\left(x_{1}=1, x_{2}=*, x_{3}=\right.$ $\left.0, x_{4}=*, \ldots, x_{n}=*\right) \in\{0,1, *\}^{n}$ is denoted by $\left(x_{1}=1, x_{3}=0\right)$. For two assignments $t_{1}, t_{2} \in\{0,1\}^{n}$ to $X$, the Hamming distance $d$ between $t_{1}$ and $t_{2}$ is $d\left(t_{1}, t_{2}\right)=\left|\left\{i \in[n]: t_{1}(i) \neq t_{2}(i)\right\}\right|$. We extend this notion to partial assignments as follows ${ }^{1}$ : for two partial assignments $t_{1}, t_{2} \in\{0,1, *\}^{n}$, the Hamming distance $d$ between $t_{1}$ and $t_{2}$ is

$$
d\left(t_{1}, t_{2}\right) \stackrel{\text { def }}{=}\left|\left\{i \in[n]: \begin{array}{l}
t_{1}(i) \neq *, t_{2}(i) \neq *, \text { and } \\
t_{1}(i) \neq t_{2}(i)
\end{array}\right\}\right|
$$

Given a partial assignment $t$, we simplify $\varphi$ in the standard way, that is, eliminating any clause from $\varphi$ if a literal of the clause is assigned 1 under $t$, and eliminating any literal from $\varphi$ if the literal is assigned 0 under $t$. The resulting formula is denoted by $\left.\varphi\right|_{t}$. For later use, we present a typical algorithm for $k$-SAT, denoted by simple-sat, in Fig. 1 below.

Proposition 2.1. Given a $k$-CNF formula $\varphi$, the running time of simple-sat $(\varphi)$ is $O\left(\beta_{k}^{n}\right)$ for some constant $\beta_{k}<2$ depending only on $k$.

As shown in section 2 of [11], $\beta_{k}$ is the largest real number $x>0$ that satisfies $x^{k}-x^{k-1}-\cdots-x^{2}-x-1=0$. (For example, $\beta_{3}=1.840$.)

We slightly modify this algorithm for our purpose. First, we omit the second "return YES" from the algorithm, that is, we just run simple-sat $\left(\left.\varphi\right|_{t}\right)$ for each partial assignment $t \in S$. Second, we therefore omit the second

\footnotetext{
${ }^{1}$ It might be better to give it another term since the extension is no longer "distance": it does not satisfy the triangle inequality.
} 


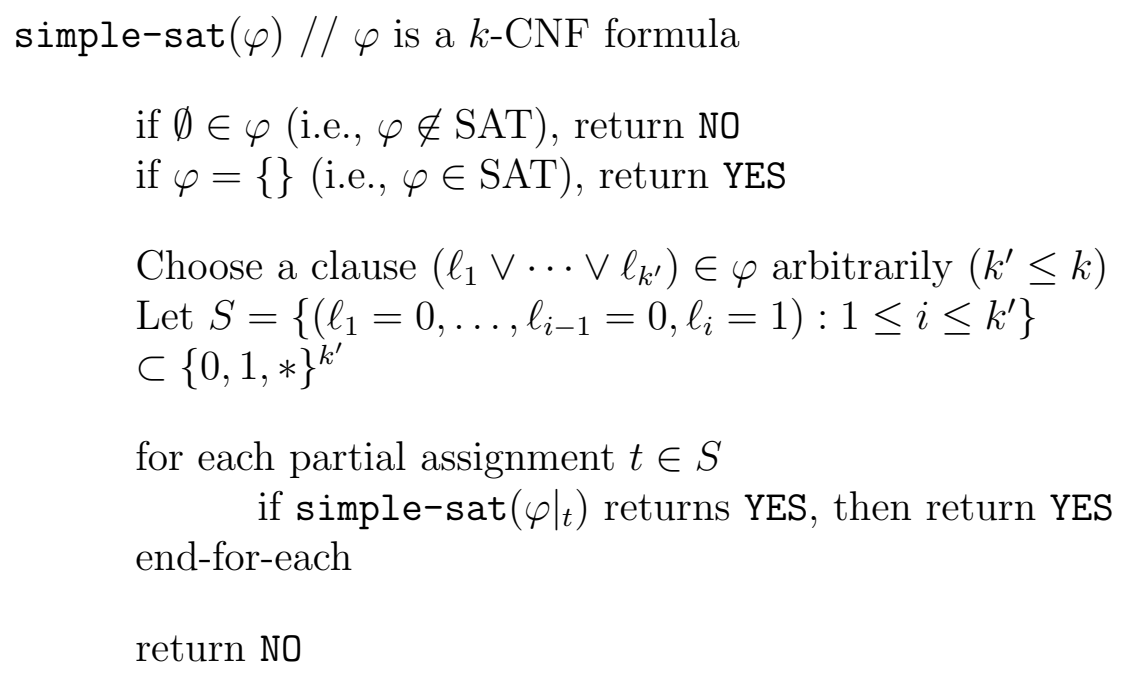

Figure 1: A simple backtracking algorithm for $k$-SAT

"return NO" from the algorithm. Note that Proposition 2.1 also holds for this modified algorithm. This modification comes from our strategies for solving CONNkSAT: we enumerate all satisfying partial assignments. In what follows, we call this modified algorithm simple-sat.

Given a $k$-CNF formula, a binary decision diagram is constructed by the execution of simple-sat $(\varphi)$. It is viewed as a rooted binary tree shown in Fig. 2: we only depict one part of the binary tree, where a recursive call of $\operatorname{simple-\operatorname {sat}}\left(\left.\varphi\right|_{t}\right)$ with $t=\left(l_{1}=0, \ldots, l_{k^{\prime}-1}=0, l_{k^{\prime}}=1\right)$ for some $k^{\prime} \leq k$ is executed. In such a binary tree, each non-leaf vertex represents a variable, and each edge is labelled with 0 or 1 . Alternatively, in such a representation, every vertex can be viewed as a partial assignment. The depth of a vertex $v$ in a binary tree is the number of ancestors of $v$.

Given a $k$-CNF formula, let $T_{\varphi}$ be the rooted binary tree obtained by running simple-sat $(\varphi)$. Let $\mathrm{SAT}_{\varphi}$ be the set of leaves of $T_{\varphi}$ that satisfy $\varphi$. We alternatively view $\mathrm{SAT}_{\varphi}$ as the set of partial satisfying assignments. For simplicity, we assume that every leaf of $T_{\varphi}$ corresponds to a partial satisfying assignment so that $\mathrm{SAT}_{\varphi}$ is exactly the set of leaves of $T_{\varphi}$. This is because such a tree is constructed by erasing from $T_{\varphi}$ all sub-trees every leaf of which are not satisfying assignments. Moreover, this construction is done in time $O\left(\beta_{k}^{n}\right)$, where $\beta_{k} \leq 2-\epsilon_{k}$ for our target bound $O\left(\left(2-\epsilon_{k}\right)^{n}\right)$. Then, we note the following two facts about $\mathrm{SAT}_{\varphi}$. 


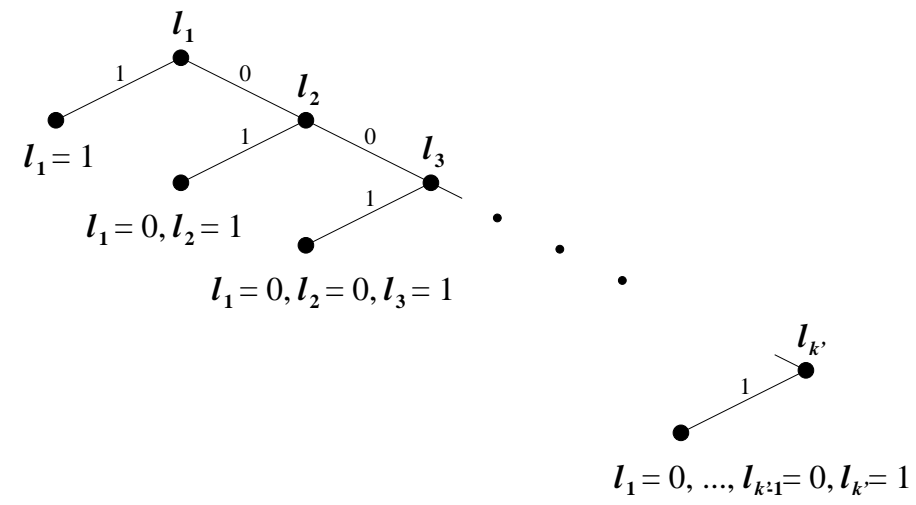

Figure 2: A binary tree

Note 2.2. For any pair of distinct vertices $u, v \in S A T_{\varphi}, d(u, v) \geq 1$.

Note 2.3. The vertex set $S A T_{\varphi}$ is a partition of the set of all satisfying assignments of $\varphi$, that is, for any satisfying assignment $t \in\{0,1\}^{n}$ of $\varphi$, there is a unique vertex $v \in S A T_{\varphi}$ (i.e., $v$ is a satisfying partial assignment) such that $d(t, v)=0$.

Given a $k$-CNF formula $\varphi$ over $n$ variables, let $\mathrm{SAT}_{\varphi}$ be as above, and let $H_{\varphi}$ be the graph induced from the $n$ dimensional hypercube by $\operatorname{SAT}_{\varphi}$. The solution space induced by $S \subset S A T_{\varphi}$ is the graph induced from $H_{\varphi}$ by $S$, that is, by $\left\{t \in\{0,1\}^{n}: \exists s \in S[d(s, t)=0]\right\}$. We here note the following two facts, which are easily shown.

Note 2.4. The solution space induced by a single vertex of $S A T_{\varphi}$ is connected.

Note 2.5. Let $v_{1}, v_{2}$ be distinct vertices of $S A T_{\varphi}$. Suppose that $d\left(v_{1}, v_{2}\right)=1$. Then, the solution space induced by $\left\{v_{1}, v_{2}\right\}$ is connected.

Given a $k$-CNF formula $\varphi$ over $n$ variables, the connectivity problem which we study, denoted by CONNkSAT, is to ask if the graph $H_{\varphi}$ is connected.

Theorem 2.6 (Gopalan et al. [6]). CONNkSAT is PSPACE-complete for $k \geq 3$. On the other hand, CONNkSAT is in $P$ for $k \leq 2$. 


\section{An Exact Algorithm for CONNkSAT}

We present an exact algorithm for CONN $k \mathrm{SAT}$, and show the running time is $O\left(\left(2-\epsilon_{k}\right)^{n}\right)$ for some constant $\epsilon_{k}>0$. The algorithm, denoted by $\operatorname{conn}-\operatorname{sat}(\varphi)$ given a $k$-CNF formula $\varphi$, is shown in Fig. 3 , where $\beta_{k}$ is the constant specified in the preliminary section.

$$
\begin{aligned}
& \operatorname{conn}-\operatorname{sat}(\varphi) \quad / / \text { parameter } \alpha \text { is a real number that satisfies } \\
& \left(2 \beta_{k}\right)^{\alpha n}=\beta_{k}^{n}
\end{aligned}
$$

Run simple-sat $(\varphi)$

Let $T_{\varphi}$ and $\mathrm{SAT}_{\varphi}$ be as defined above

Let $V_{\varphi}=V\left(T_{\varphi}\right)$ be the set of vertices of $T_{\varphi}$,

Let $E_{\varphi}=\emptyset$

Construct an undirected graph $G_{\mathrm{SAT}}=\left(\operatorname{SAT}_{\varphi}, E_{\varphi}\right)$

as follows:

(1) for each pair of vertices $u, v \in \operatorname{SAT}_{\varphi}$ with depth

at most $(1-\alpha) n$

if $d(u, v)=1$, then add $(u, v)$ to $E_{\varphi}$

(2) for each $u \in \operatorname{SAT}_{\varphi}$ with depth more than $(1-\alpha) n$

Visit $v \in V_{\varphi}$ in the depth-first search manner starting from the root of $T_{\varphi}$ so that

if $d(u, v) \geq 2$, then do not visit vertices

which are descendants of $v$ any longer

else if $v \in \operatorname{SAT}_{\varphi}$ and $v \neq u$, then add $(u, v)$

to $E_{\varphi}$

if $G_{\mathrm{SAT}}=\left(\mathrm{SAT}_{\varphi}, E_{\varphi}\right)$ is connected, output YES,

else output NO

Figure 3: An exact algorithm for CONNkSAT

The idea of this algorithm is to enumerate all satisfying partial assignments using simple-sat, and to construct a graph over those assignments such that there is an edge between two satisfying partial assignments if and only if the Hamming distance between them is exactly one. (Recall Note 2.2 that $d(u, v) \geq 1$ for any pair of distinct vertices $u, v \in \operatorname{SAT}_{\varphi}$.) Then, we can 
easily check the connectivity of the graph in linear time with respect to its size, i.e., the number of vertices and edges of the graph.

Before proceeding to the formal analysis of the algorithm, we shall give intuition on how to upper-bound the running time. The crucial point is to show that the size of the constructed graph is at most $O\left((2-\epsilon)^{n}\right)$ for some constant $\epsilon>0$. We can see that the number of vertices is at most $O\left(\beta_{k}^{n}\right)$ by Proposition 2.1 since the set of vertices consists of leaves in the tree obtained by running simple-sat. However, it is not obvious to bound the number of edges. One trivial upper bound, the square of the number of vertices, may exceed $2^{n}$. (For example, $\left(\beta_{3}^{n}\right)^{2}>3.385^{n}$.) Another trivial upper bound, the sum of the degree of the vertices, may also exceed $2^{n}$ since each vertex corresponds to a partial assignment and can have exponentially large degree (up to $2^{s}$ where $s$ is the number of *'s in the assignment).

Our key observation is the following two facts; (i) the number of "shallow" vertices is small and (ii) the degree of "deep" vertices is small. Here shallow and deep mean the depth of a vertex in the tree associated with the execution of simple-sat. Fact (i) essentially follows from Proposition 2.1 and fact (ii) holds since a deep vertex corresponds to an assignment with few *'s. Then we classify edges into two types, (shallow, shallow) and (deep,any). The number of the former and the latter types can be bounded by $O\left((2-\epsilon)^{n}\right)$ using the square of the number of the shallow vertices and the sum of the degree of the deep vertices, respectively.

Now we proceed to the formal analysis.

Lemma 3.1. Given a $k$-CNF formula $\varphi$, let $G_{S A T}=\left(S A T_{\varphi}, E_{\varphi}\right)$ be the final $G_{S A T}$ obtained by constructing $E_{\varphi}$. Let $v_{1}, v_{2} \in S A T_{\varphi}$ be distinct vertices. Then,

$$
d\left(v_{1}, v_{2}\right)=1 \Longleftrightarrow\left(v_{1}, v_{2}\right) \in E_{\varphi} .
$$

Proof. Note first that $d\left(v_{1}, v_{2}\right) \geq 1$, which comes from Note 2.2. It is easy to see that $(u, v) \in E_{\varphi}$ implies $d(u, v)=1$ since our algorithm adds an edge $(u, v)$ to $E_{\varphi}$ only if $d(u, v)=1$.

Suppose that $d(u, v)=1$. We see that it means our algorithm adds an edge $(u, v)$ to $E_{\varphi}$ because of the following observation: if $d(u, v)=1$, then $d(u, w) \leq 1$ for any ancestor $w$ of $v$.

Lemma 3.2. Given a $k$-CNF formula $\varphi$, let $G_{S A T}=\left(S A T_{\varphi}, E_{\varphi}\right)$ be the final $G_{S A T}$ obtained by constructing $E_{\varphi}$. Then,

$$
\varphi \in C O N N k S A T \Longleftrightarrow G_{S A T} \text { is connected. }
$$


Proof. We first consider the case of $\left|\operatorname{SAT}_{\varphi}\right| \leq 1$. In this case, it is obvious that $G_{\mathrm{SAT}}=\left(\operatorname{SAT}_{\varphi}, E_{\varphi}\right)$ is connected. Moreover, $\varphi \in \mathrm{CONN} k \mathrm{SAT}$ holds because of Note 2.4. Thus, this lemma holds for $\left|\operatorname{SAT}_{\varphi}\right| \leq 1$.

Next, we assume that $\left|\mathrm{SAT}_{\varphi}\right| \geq 2$. Suppose that $G_{\mathrm{SAT}}$ is connected. We will show that any pair of two satisfying assignments of $\varphi$ is connected on $H_{\varphi}$. Let $t_{1}, t_{2} \in\{0,1\}^{n}$ be distinct satisfying assignments of $\varphi$. Let $v_{1} \in \operatorname{SAT}_{\varphi}$ (resp. $v_{2} \in \mathrm{SAT}_{\varphi}$ ) be a vertex of $G_{\mathrm{SAT}}$ corresponding to $t_{1}$ (resp. $\left.t_{2}\right)$, that is, $d\left(v_{1}, t_{1}\right)=0$ (resp. $\left.d\left(v_{2}, t_{2}\right)=0\right)$. From Note 2.3 , there is such a vertex which is unique. We may assume $v_{1} \neq v_{2}$ since otherwise it is the same as the case of $\left|\mathrm{SAT}_{\varphi}\right| \leq 1$. Since $G_{\mathrm{SAT}}$ is connected, there is a path between $v_{1}$ and $v_{2}$ (on $G_{\mathrm{SAT}}$ ). Consider any pair of adjacent vertices on the path, say, $u_{1}, u_{2} \in \mathrm{SAT}_{\varphi}$, From the previous lemma, $d\left(u_{1}, u_{2}\right)=1$ since $\left(u_{1}, u_{2}\right) \in E_{\varphi}$. Moreover, from Note 2.5, the solution space of $H_{\varphi}$ induced by $\left\{u_{1}, u_{2}\right\}$ is connected. Applying this argument repeatedly to every pair of adjacent vertices on the path, we see that the solution space of $H_{\varphi}$ induced by all vertices on the path is connected, and hence $t_{1}$ and $t_{2}$ are connected on $H_{\varphi}$. This holds for any pair of two satisfying assignments of $\varphi$. Thus, we conclude $\varphi \in \mathrm{CONN} k \mathrm{SAT}$.

Suppose that $\varphi \in \mathrm{CONN} k \mathrm{SAT}$. We will show that any pair of two vertices of $\mathrm{SAT}_{\varphi}$ is connected on $G_{\mathrm{SAT}}$. Let $v_{1}, v_{2} \in \mathrm{SAT}_{\varphi}$ be distinct vertices of $G_{\mathrm{SAT}}$. Let $t_{1}$ (resp. $t_{2}$ ) be an arbitrary satisfying assignment of $\varphi$ such that $d\left(t_{1}, v_{1}\right)=0\left(\operatorname{resp} . d\left(t_{2}, v_{2}\right)=0\right)$. Since $\varphi \in \mathrm{CONN} k \mathrm{SAT}$, there exists a path $t_{1}=a_{0} \rightarrow a_{1} \rightarrow \ldots \rightarrow a_{\ell}=t_{2}$ on $H_{\varphi}$. Consider any pair of $a_{i}$ and $a_{i+1}$. There are two cases: (1) there is a vertex $u \in \operatorname{SAT}_{\varphi}$ such that $d\left(a_{i}, u\right)=d\left(a_{i+1}, u\right)=0$, and (2) there are distinct vertices $u_{1}, u_{2} \in \operatorname{SAT}_{\varphi}$ such that $d\left(a_{i}, u_{1}\right)=d\left(a_{i+1}, u_{2}\right)=0$. Consider the second case. (We do not need to care for the first case.) Since $d\left(a_{i}, a_{i+1}\right)=1$, we must have $d\left(u_{1}, u_{2}\right)=1$. (We do not have $d\left(u_{1}, u_{2}\right)=0$ since $u_{1} \neq u_{2}$.) From the previous lemma, it means $\left(u_{1}, u_{2}\right) \in E_{\varphi}$. Applying this argument repeatedly to every pair of adjacent vertices on the path, we see that $v_{1}$ and $v_{2}$ are connected on $G_{\mathrm{SAT}}$. This holds for any pair of two vertices of $\operatorname{SAT}_{\varphi}$. Thus, we conclude that $G_{\varphi}$ is connected.

From this lemma, we conclude that the output of $\operatorname{conn}-\operatorname{sat}(\varphi)$ is correct for any $\varphi$. It remains to show the upper bound on the running time of $\operatorname{conn}-\operatorname{sat}(\varphi)$.

Lemma 3.3. The running time of conn-sat $(\varphi)$ is $O\left(\left(2-\epsilon_{k}\right)^{n}\right)$ for some constant $\epsilon_{k}>0$ depending only on $k$. 
Proof. Given a $k$-CNF formula $\varphi$, let $T_{\varphi}$ be the rooted binary tree obtained by running simple-sat $(\varphi)$. Let $G_{\mathrm{SAT}}=\left(\operatorname{SAT}_{\varphi}, E_{\varphi}\right)$ be the final $G_{\mathrm{SAT}}$ obtained by constructing $E_{\varphi}$. Note here that the running time of constructing $T_{\varphi}$ is $O\left(\beta_{k}^{n}\right)$, where $\beta_{k}$ is the constant specified in the preliminary section. For showing the worst-case running time, it suffices to estimate an upper bound of $\left|E_{\varphi}\right|$. For any $\alpha: 0 \leq \alpha \leq 1$, let

$$
\begin{aligned}
& U \stackrel{\text { def }}{=}\left\{u \in \operatorname{SAT}_{\varphi}: \operatorname{depth}(u) \leq(1-\alpha) n\right\}, \\
& W \stackrel{\text { def }}{=}\left\{w \in \operatorname{SAT}_{\varphi}: \operatorname{depth}(w)>(1-\alpha) n\right\},
\end{aligned}
$$

where $\operatorname{depth}(u)$ is the depth of $u$ in $T_{\varphi}$. Then,

$$
\left|E_{\varphi}\right|=\left|E_{1}\right|+\left|E_{2}\right|, \text { where }\left\{\begin{array}{l}
E_{1}=E_{\varphi} \cap(U \times U), \\
E_{2}=\left(E_{\varphi} \cap(U \times W)\right) \cup\left(E_{\varphi} \cap(W \times W)\right) .
\end{array}\right.
$$

Claim 3.4. For any $\alpha: 0 \leq \alpha \leq 1$,

$$
\begin{aligned}
& \text { 1. }\left|E_{1}\right| \leq\left(\beta_{k}^{(1-\alpha) n}\right)^{2}\left(=\beta_{k}^{2(1-\alpha) n}\right), \\
& \text { 2. }\left|E_{2}\right| \leq \sum_{0 \leq t \leq \alpha n} \beta_{k}^{n-t}\left((n-t) 2^{t}\right) \quad\left(\leq n^{2} \cdot 2^{\alpha n} \cdot \beta_{k}^{(1-\alpha) n}\right) .
\end{aligned}
$$

Proof. The first inequality comes from the fact that the number of vertices of $T_{\varphi}$ with depth at most $(1-\alpha) n$ is at most $\beta_{k}^{(1-\alpha) n}$.

Fix $t$ with $0 \leq t \leq \alpha n$ arbitrarily. Consider an arbitrary vertex $w \in W$ with depth $n-t$. We will estimate the possible number of edges $(w, v) \in E_{\varphi}$ where $v \in \operatorname{SAT}_{\varphi}$. Let $r_{i}$ be the ancestor of $w$ at depth $i(0 \leq i<n-t)$. Let $r_{i}^{\prime}$ be the child vertex of $r_{i}$ that is not an ancestor of $w$. Let $T_{w, i}$ be the sub-tree of $T_{\varphi}$ rooted at $r_{i}^{\prime}$. Then, the number of assignments (not necessarily satisfying ones) $a \in\{0,1\}^{n}$ such that $d\left(r_{i}^{\prime}, a\right)=0$ and $d(w, a) \leq 1$ is exactly $2^{t}$ since the number of variables assigned $*$ under $w$ is $t$. Let $A \subset\{0,1\}^{n}$ be the set of those assignments. Then, the number of leaves $v$ of $T_{w, i}$ such that $d(w, v) \leq 1$ is at most $2^{t}$ since each assignment $a \in A$ corresponds to a unique leaf $v$ if $a$ is a satisfying assignment. Thus, for any $w \in W$ with depth $n-t$, the number of leaves $v$ such that $d(w, v) \leq 1$ is at most $(n-t) 2^{t}$. Since the number of vertices $w \in W$ with depth $n-t$ is at most $\beta_{k}^{n-t}$, the second inequality holds.

From this claim, we have $\left|E_{\varphi}\right| \leq \beta_{k}^{2(1-\alpha) n}+n^{2} 2^{\alpha n} \beta_{k}^{(1-\alpha) n}$ for any $0 \leq$ $\alpha \leq 1$. By fixing $\alpha$ to a constant satisfying $\beta_{k}^{2(1-\alpha) n}=2^{\alpha n} \beta_{k}^{(1-\alpha) n}$, which is 
equivalent to $\left(2 \beta_{k}\right)^{\alpha n}=\beta_{k}^{n}$, we obtain an upper bound on $\left|E_{\varphi}\right|$ as follows:

$$
\left|E_{\varphi}\right| \leq 2 \cdot \operatorname{poly}(n) \cdot 2^{\alpha n} \beta_{k}^{(1-\alpha) n} .
$$

We see that the formula on the right-hand-side is $O\left(\left(2-\epsilon_{k}\right)^{n}\right)$ for some constant $\epsilon_{k}>0$ (depending only on $k$ ) since $\beta_{k}$ is a constant less than 2 .

From Lemma 3.2, we see that our algorithm solves CONN $k$ SAT. From Lemma 3.3, we see that our algorithm runs in time $O\left(\left(2-\epsilon_{k}\right)^{n}\right)$ for some constant $\epsilon_{k}>0$. Therefore, we obtain the following theorem:

Theorem 3.5. The problem CONNkSAT is solvable in time $O\left(\left(2-\epsilon_{k}\right)^{n}\right)$ for some constant $\epsilon_{k}>0$ depending only on $k$. (For example, it is $O\left(1.914^{n}\right)$ for $k=3$.)

Lemma 3.2 actually shows that the number of connected components in $H_{\phi}$ is same as that in $G_{\mathrm{SAT}}$. Thus, we have:

Corollary 3.6. Given a $k$-CNF formula $\varphi$ over $n$ variables, the number of connected components in $H_{\varphi}$ can be computed in time $O\left(\left(2-\epsilon_{k}\right)^{n}\right)$ for the constant $\epsilon_{k}$ given in Theorem 3.5.

\section{Conclusion}

We have presented an $O\left(2-\epsilon_{k}\right)^{n}$-time exact algorithm for CONN $k$ SAT. One of our future work is to improve the analysis of the running time of our algorithm, and to obtain the upper bound $O\left(\beta_{k}^{n}\right)$ which is same as the running time of simple-sat: our bound is slightly worse than $O\left(\beta_{k}^{n}\right)$. Instead of doing that, we may be able to reduce the running time just by replacing simple-sat with a more sophisticated backtrack-type algorithm $A$ that satisfies the following: all leaves of the rooted binary tree constructed by $A$ constitute a partition of all satisfying assignments. However, we encounter the same problem as above: we cannot derive the running time as much as that of $A$ from our analysis.

\section{References}

[1] R. Bellman, "Dynamic programming treatment of the travelling salesman problem," Journal of the ACM, 9(1):61-63, 1962. 
[2] A. Björklund, T. Husfeldt, P. Kaski, and M. Koivisto, "The travelling salesman problem in bounded degree graphs," In Proc. ICALP 2008 part I, pp. 198-209, 2008.

[3] A. Björklund, T. Husfeldt, P. Kaski, and M. Koivisto, "Fourier meets Möbius: Fast subset convolution," In Proc. STOC 2007, pp. 67-74, 2007.

[4] A. Björklund and T. Husfeldt, "Inclusion-exclusion algorithms for counting set partitions," In Proc. FOCS 2006, pp. 575-582, 2006.

[5] C. Calabro, "The exponential complexity of satisfiability problems," $\mathrm{Ph}$. D. thesis, University of California, San Diego, 2009.

[6] P. Gopalan, P. G. Kolaitis, E. N. Maneva, and C. H. Papadimitriou, "The connectivity of Boolean satisfiability: Computational and structural dichotomies," SIAM J. Comput., 38(6):2330-2355, 2009.

[7] M. Held and R. M. Karp, "The traveling-salesman problem and minimum spanning trees," Operations Res., 18(6):1138-1162, 1970.

[8] K. Iwama and S. Tamaki, "Improved upper bounds for 3-SAT," In Proc. SODA 2004, pp. 328-329, 2004.

[9] M. Koivisto, "An $O\left(2^{n}\right)$ algorithm for graph coloring and other partitioning problems via inclusion-exclusion," In Proc. FOCS 2006, pp. 583-590, 2006.

[10] K. Kutzkov, "New upper bound for the \#3-SAT problem," Inf. Process. Lett., 105(1):1-5, 2007.

[11] B. Monien and E. Speckenmeyer, "Solving satisfiability in less than $2^{n}$ steps," Discrete Applied Mathematics, 10:287-295, 1985.

[12] K. Makino, S. Tamaki, M. Yamamoto, "On the Boolean connectivity problem for Horn relations," Discrete Applied Mathematics, to appear.

[13] R. Paturi, P. Pudlák, M. E. Saks, and F. Zane, "An improved exponential-time algorithm for $k$-SAT," J. ACM, 52(3):337-364, 2005.

[14] R. Paturi, P. Pudlák, and F. Zane, "Satisfiability coding lemma," Chicago J. Theor. Comput. Sci., 1999. 
[15] D. Rolf, "Improved bound for the PPSZ/Schöning-algorithm for 3-SAT," JSAT, 1:111-122, 2006.

[16] U. Schöning, "A probabilistic algorithm for $k$-SAT based on limited local search and restart," Algorithmica, 32(4):615-623, 2002.

[17] U. Schöning, "Algorithmics in exponential time," In Proc. STACS 2005, pp. 36-43, 2005.

[18] R. Williams, "Algorithms for quantified Boolean formulas," In Proc. SODA 2002, pp. 299-307, 2002.

[19] R. Williams, "A new algorithm for optimal constraint satisfaction and its implications," In Proc. ICALP 2004, pp. 1227-1237, 2004.

[20] G. J. Woeginger, "Exact algorithms for NP-hard problems: a survey," In Combinatorial Optimization (Edmonds Festschrift), LNCS 2570, pp. 185-207, 2003. 